\title{
EFECTO DE DISTINTOS SISTEMAS DE PRODUCCIÓN Y FORMAS DE SUJECIÓN SOBRE LAS PÉRDIDAS POSCOSECHA EN ACELGA (BETA VULGARIS L. VAR. CICLA L.)
}

\author{
Grasso, R. ${ }^{1} ;$ Rotondo, R. ${ }^{1 ;}$ Ortiz Mackinson, M. ${ }^{1 ;}$ Mondino, M. ${ }^{1,3}$; \\ Calani, P. ${ }^{1}$; Balaban, D. ${ }^{1}$; Vita Larrieu, E..$^{1,4}$ \& Torres, P. ${ }^{2}$
}

\begin{abstract}
RESUMEN
El trabajo se realizó en la FCA-UNR en otoño, invierno y primavera, con el objetivo de evaluar el efecto de los sistemas de producción, forma de sujeción y días de almacenamiento sobre el rendimiento y las pérdidas poscosecha. El ensayo se realizó teniendo en cuenta tres factores: 1) sistemas de producción: invernadero (I), media sombra (MS), manta flotante (MF) y campo (C), 2) formas de sujeción: manojo (A) y granel $(\mathrm{G})$ y 3 ) días de almacenamiento. Las variables medidas fueron: rendimiento, materia seca, pérdida de peso por descarte y por agua e índice de color. En las tres épocas, I obtuvo el mayor rendimiento y menor contenido de materia seca. I y MF obtuvieron las menores pérdidas de peso por descarte en otoño; en invierno y otoño aumentan con los días de almacenamiento. I y MF registraron las menores pérdidas de peso por agua en otoño en los días 3 y 6 de almacenamiento. La acelga mostró una tendencia hacia el verde amarillento en MF en otoño, C en invierno e I en primavera, en todos los días de almacenamiento.
\end{abstract}

Palabras claves: invernadero, media sombra, manta flotante, atado, granel.

1.- Cátedra de Cultivos Intensivos, Horticultura. Facultad de Ciencias Agrarias (UNR). C.C. 14. (S2125ZAA) Zavalla, provincia de Santa Fe. Email: rgrasso@unr.edu.ar

2.-Cátedra de Ecología. FCA (UNR)

3.- AER INTA Arroyo Seco.

4.- AER INTA Pago de los Arroyos.

Manuscrito recibido el 12 de marzo de 2018 y aceptado para su publicación el 15 de agosto de 2018.

Grasso, R.; Rotondo, R.; Ortiz Mackinson, M.; Mondino, M.; Calani, P.; Balaban, D.; Vita Larrieu, E. \& Torres, P. Efecto de distintos sistemas de producción y formas de sujeción sobre las pérdidas poscosecha en acelga (Beta vulgaris l. Var. Cicla l.). FAVE - Ciencias Agrarias 17 (1): 21-33.

CC BY-NC-SA 4.0 cC)(3) (2) 


\section{ABSTRACT \\ Effect of different production systems and fastening methods on postharvest losses in swiss chard (Beta Vulgaris I. Var. Cicla I.)}

This work, carried out in the College of Agricultural Sciences, UNR, in autumn, winter and spring with the objective of evaluating the effect the effect evaluates the effect of production systems, fastening methods and days of storage on yield and postharvest losses on Swiss chard along three seasons. The trial was carried out taking into account three factors: 1) Production systems: greenhouse (I), shade cloth (MS), frost protection fleece fabric (MF), and open field (C); 2) fastening method: bunches (A) and no fastening $(G) ; 3)$ storage days. The variables measured were: yield, dry matter, weight loss by discarding, weight loss by water, and color index. In all three seasons, I obtained the highest yield and lowest dry matter content. I and MF obtained the least weight loss by discarding in autumn; in winter and autumn they increase with the days of storage. I and MF recorded the lowest weight losses per water in autumn on days 3 and 6 of storage. Chard showed a tendency towards yellowish green in MF in autumn, $\mathrm{C}$ in winter and I in spring, in all days of storage.

Key words: greenhouse, shade cloth, frost protection fleece fabric, tied, bulk.

\section{INTRODUCCIÓN}

La acelga es una hortaliza que pertenece a la familia de las Quenopodiáceas y cuyo consumo está muy difundido en los países mediterráneos. En Argentina se produce en los cinturones hortícolas que rodean los principales centros urbanos de todo el país (2), siendo dos los destinos de la producción, el consumo en fresco y la industrialización (congelado, enlatado, deshidratado, etc.). El Cinturón Hortícola de Rosario es el más importante de la provincia de Santa $\mathrm{Fe}$, no sólo por su producción sino también por la actividad comercial. La misma se realiza principalmente a través de dos mercados concentradores que abastecen a más de 2,5 millones de habitantes (3). En esta región se cultivan una gran diversidad de hortalizas, entre los que se destaca la acel- ga, ocupando el cuarto lugar en importancia en cuanto a superficie cultivada con 322 hectáreas (5).

Las hortalizas son altamente perecederas debido a su elevada transpiración, actividad respiratoria, susceptibilidad a los daños físicos y enfermedades, que pueden producir pérdidas, daños y una reducción de la calidad organoléptica si no son controladas adecuadamente. La forma de sujetar algunas hortalizas en manojos, provocan daños que disminuyen la calidad y la posibilidad de comercialización (15). Se estiman considerables las pérdidas en cantidad y calidad de la producción anual en el país, que ocurren entre la cosecha y el consumo. Estas pérdidas son ocasionadas básicamente por condiciones de producción inadecuadas, causas mecánicas, desórdenes fisiológicos y enfermedades causadas por microorganismos (13). 
La composición y calidad de los productos hortícolas generalmente es influenciada por muchos factores de precosecha. Estos factores que influyen sobre la calidad son muy diversos y están interrelacionados entre sí. Unos dependen intrínsecamente de la propia planta y son la integración del flujo de energía, agua y nutrientes, otros son de tipo genético, ambiental y de cultivo (18).

El uso de cubiertas con materiales plásticos en la producción de hortalizas tiene diferentes propósitos. En regiones templadas, son usadas principalmente durante la temporada fría para conservar el calor, estimular la germinación, producir primicias, proteger las plantas de los daños por heladas y mejorar la calidad de los cultivos. Otros efectos benéficos que pueden esperarse son, mantener la textura del suelo, proteger al cultivo del ataque de pájaros y otras plagas. En épocas de altas temperaturas, los materiales deben tener adecuada permeabilidad al aire y la humedad para prevenir la excesiva temperatura bajo la cubierta (8).

Según Jensen (10), debido al control de los factores de producción, el cultivo de vegetales bajo cubierta es normalmente más confiable. Las amplias y erráticas fluctuaciones que se producen en los cultivos a campo pueden ser reducidas significativamente. Mashego (12), comenta que un problema significativo que atraviesa la agricultura mundial es la variación de los rendimientos de los cultivos de un año a otro. Esto se debe a la variación del estrés por sequía o inundación, alta velocidad del viento y bajas o altas temperaturas. Los daños que causan las condiciones de estrés pueden producir desórdenes fisiológicos en los cultivos.

En los últimos años el uso de sombreaderos (malla media sombra), agrotextiles e invernaderos ha tenido una gran difusión entre los productores en nuestro país. Estos sistemas permiten el aumento de la calidad comercial y continuidad de la producción durante todo el año, presentando así un valor estratégico en el esquema de comercialización y vida poscosecha. Los sombreaderos son estructuras construidas con el objetivo de reducir la radiación, la temperatura a nivel de hojas, la evapotranspiración, aumentar la humedad relativa del ambiente y regular la humedad a nivel del suelo. Tienen un importante desarrollo, dado la reducción de los costos de inversión respecto a los invernaderos y la posibilidad de lograr mejores condiciones de cultivo en verano. En el sur de la provincia de Santa Fe, en época estival, su utilización es una alternativa para que los productores puedan competir con la producción proveniente de otros lugares del país, donde el verano es más benigno (3).

Las mantas flotantes, también llamadas agrotextiles, son plásticos (polietileno, poliéster, polipropileno, etc.) de fibras no tejidas, que constituyen un material muy liviano y de buena porosidad (3). Permiten el paso de más del $85 \%$ de la radiación solar. Las mismas se colocan directamente sobre el cultivo, en general en cultivos de hoja, sin ningún tipo de estructura de soporte, reduciendo los costos iniciales de inversión. Este material protege a las plantas contra vientos y logra una diferencia térmica de aproximadamente $2{ }^{\circ} \mathrm{C}$ entre el interior de la manta y el aire libre. Todos los estadios de crecimiento y desarrollo de los vegetales presentan sensibilidad a las temperaturas. El manejo de las temperaturas controla la tasa de desarrollo de los cultivos. Las hortalizas generan mayores áreas foliares, incrementan el número de hojas y el peso con respecto a las no cubiertas (14). Además, los vegetales cultivados bajo una cubierta de agrotextil, crecen más rápidamente que los no cubiertos (7). En algunos casos, se 
utilizan como barrera para reducir la entrada de insectos al cultivo y como protección contra las heladas (19).

Los invernaderos son estructuras cubiertas de polietileno que permiten lograr un incremento del rendimiento comercial y calidad, acortamiento del ciclo productivo, continuidad de la producción y protección de heladas, lluvias, granizo, entre otros. En nuestra región, la producción de hortalizas en invernadero se inició a principios de la década de 1980-1990 (1).

En los procesos productivos y durante la comercialización de las hortalizas de hoja, las pérdidas de peso y el descarte significan una disminución en la rentabilidad. A lo largo de este complejo proceso, la calidad comercial está determinada por la variación del aspecto. Resulta necesario realizar estudios para determinar la influencia de los sistemas de producción sobre el rendimiento y el comportamiento poscosecha de acelga.

El objetivo del presente trabajo fue evaluar el efecto de los distintos sistemas de producción, forma de sujeción y días de almacenamiento sobre el rendimiento, las pérdidas poscosecha e índice de color en otoño, invierno y primavera.

\section{MATERIALES Y MÉTODOS}

El trabajo se realizó en el Campo Experimental de la Facultad de Ciencias Agrarias de la Universidad Nacional de Rosario (Zavalla, Santa Fe, $33^{\circ} 01^{\prime} \mathrm{S}$ y $60^{\circ} 53^{\prime} \mathrm{O}$ ) Argentina. El cultivo se desarrolló en un suelo Argiudol Típico de la serie Roldán, cuyas características de 0-0,20 m fueron: carbono $1,71 \%$, materia orgánica $2,95 \%$, nitratos $49,89 \mathrm{ppm}$, fósforo asimilable 63,96 $\mathrm{mg} \mathrm{kg}^{-1}$. El agua utilizada para el riego complementario tenía un $\mathrm{pH}$ de 7,51 ; CIC de 22,7 meq $100 \mathrm{~g}^{-1}$ y conductividad eléctrica de $0,15 \mathrm{dS} \mathrm{m}^{-1}$.

Previo a la sistematización del terreno, se incorporó cáscara de arroz el 17/03/16 en dosis de $110 \mathrm{~m}^{3} \mathrm{ha}^{-1}$. Para la preparación de la tierra se realizó una labor de cincel en profundidad y una de disco de doble acción. El terreno se sistematizó en platabandas de $1 \mathrm{~m}$ de ancho, cubiertas con polietileno bicapa, separadas por pasillos de $0,40 \mathrm{~m}$ (Figura 1). El sistema de riego utilizado fue localizado (goteo) con laterales de 200 micrones de espesor, difusores a $0,20 \mathrm{~m}$, densidad de 7 goteros $\mathrm{m}^{-2}$, con una intensidad de 5 $\mathrm{mm} \mathrm{m} \mathrm{m}^{-2} \mathrm{hs}^{-1}$. La siembra se realizó en multimacetas plásticas de 200 celdas y el trasplante, en 4 hileras sobre las platabandas con dos laterales de goteo y una densidad de $7,14 \mathrm{pl} \mathrm{m}^{-2}$. La variedad implantada fue D'Ampuis de Clause (Alliance Semillas). Se efectuó fertirriego en dosis de $90 \mathrm{~kg} \mathrm{ha}^{-1}$ de N, $30 \mathrm{~kg} \mathrm{ha}^{-1}$ de $\mathrm{P}_{2} \mathrm{O}_{5}$ y $120 \mathrm{~kg} \mathrm{ha}^{-1}$ de $\mathrm{K}_{2} \mathrm{O}$ en cada cultivo. La superficie en cada sistema de producción fue de $30 \mathrm{~m}^{2}$.

La cosecha consistió en un deshoje, que representa una práctica habitual en la zona. Para ello se consideró el momento óptimo en función del desarrollo vegetativo y se llevó a cabo en 3 momentos coincidentes con una cosecha de otoño, de invierno y de primavera (Tabla 1).

Se analizaron tres factores:

1) Sistemas de producción

- Invernadero de madera, a dos aguas, con polietileno LDT de $100 \mu$ (I)

- Malla media sombra al 35\% (MS)

- Manta flotante agrotextil (MF)

- Campo (C) 


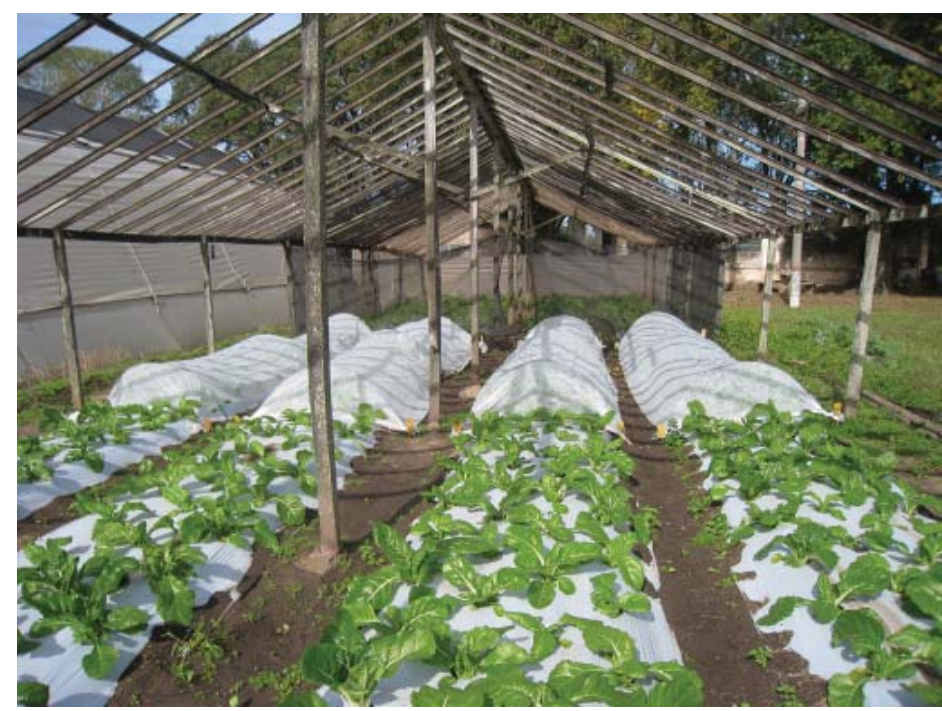

Figura 1: Vista de los sistemas de producción, platabanda y cultivo de acelga

Tabla 1: Fecha de siembra, trasplante y cosecha de las tres épocas de cultivo

\begin{tabular}{|c|c|c|c|}
\hline \multirow{2}{*}{ Fecha } & \multicolumn{3}{|c|}{ Época de cultivo } \\
\cline { 2 - 4 } & Otoño & Invierno & Primavera \\
\hline Siembra & \multicolumn{3}{|c|}{$28 / 02 / 16$} \\
\hline Transplante & $07 / 04 / 16$ \\
\hline $\begin{array}{c}\text { Cosecha } \\
\text { (deshoje) }\end{array}$ & $28 / 06 / 16$ & $16 / 08 / 16$ & $20 / 09 / 16$ \\
\hline
\end{tabular}

2) Formas de sujeción

- Manojo de hojas atado con cinta de papel adhesiva de 2 $\mathrm{cm}$ de ancho, de un peso aproximado de $500 \mathrm{~g}$ (A)

- Hojas a granel, con un peso equivalente al manojo $(\mathrm{G})$

3) Días de almacenamiento

- El material luego de la cosecha se conservó en cámara frigorífica a $3{ }^{\circ} \mathrm{C}$ y $99 \%$ H.R. Se realizaron mediciones en los días 0, 3, 6 y 9 de almacenamiento.
Las variables analizadas fueron:

Rendimiento $\left(\mathrm{kg} \mathrm{m}^{-2}\right)$ : el material vegetal cosechado respetó los parámetros de calidad comercial, seleccionando hojas de acelga túrgidas, tiernas, limpias, sin daños mecánicos, libres de plagas y enfermedades, con medidas promedio de $0,17 \mathrm{~m}$ de pecíolo y láminas de 0,23 m de largo, color verde brillante. La unidad experimental fueron hojas cosechadas de parcelas de $1 \mathrm{~m}^{2}$, pesadas con balanza digital Systel Bummer $\pm 1 \mathrm{~g}$. 


\section{R. Grasso et al.}

Materia seca (\%): una muestra del material cosechado se colocó en estufa a $60{ }^{\circ} \mathrm{C}$, secadas hasta peso constante. El porcentaje de peso seco se refirió al peso fresco inicial.

Luego de la cosecha se acondicionaron las muestras de acuerdo con la forma de sujeción y se hidrataron mediante inmersión en agua a $19{ }^{\circ} \mathrm{C}$ y $7,2 \mathrm{pH}$, durante 5 minutos. Posteriormente, el material vegetal se dejó escurrir durante 15 minutos; se registró el peso inicial (valor del día 0) y se almacenó en cámara durante los días del experimento a $3{ }^{\circ} \mathrm{C}$ y $99 \%$ H.R.

Pérdida de peso por descarte (\%): fueron consideradas en esta pérdida a las hojas que presentaron daños por roturas, amarillamiento, deshidratación, con podredumbres y base de pecíolos con pardeamiento. La cuantificación de esta pérdida se realizó mediante pesada gravimétrica con balanza digital Systel Bumer \pm 1 g. Posteriormente se procedió a retirar de cada muestra aquellas partes que presentaron algunos de los defectos antes mencionados, los que fueron pesados y considerados como pérdida o descarte. Esta pérdida fue expresada como porcentaje respecto al peso de la muestra completa antes del descarte, de ese día de evaluación.

Pérdida de peso por agua (\%): en función a la evaporación del agua libre y deshidratación por transpiración. En cada medición se pesó cada muestra con balanza digital Systel Bumer \pm 1 g, considerándose la variación de peso que sufrió el producto con respecto a la medición anterior, luego de haberse retirado el descarte respectivo.

Índice de color: debido a que la vida útil de la acelga está determinada por la variación de color hacia tonalidades claras (verdes claros o amarillentos) el índice de color es una medida válida a utilizar para determinar su vida útil.
La determinación del índice de color (IC*) fue obtenida por la expresión (1) donde $\mathrm{L}^{*}, \mathrm{a}^{*} \mathrm{y} \mathrm{b}^{*}$ son los parámetros del sistema color CIELab (21) que fueron determinados con colorímetro Chroma Meter CR300. El parámetro a* corresponde a la variación entre el rojo $\left(\mathrm{a}^{+}\right)$y el verde $\left(\mathrm{a}^{\mathrm{a}}\right)$, mientras que el parámetro $b^{*}$ representa la variación entre el color amarillo $\left(\mathrm{b}^{+}\right)$y el color azul $\left(b^{-}\right)$. El parámetro $L^{*}$, que representa la luminosidad del color, puede tomar valores en una escala que van del 0 (color blanco) al 100 (color negro) (20). El valor de los parámetros de color de las muestras se obtuvo promediando las medidas obtenidas de tres lecturas en el limbo de tres hojas de acelga, de cada repetición.

$$
I C^{*}=\frac{a^{*} \times 1.000}{L^{*} \times b^{*}}
$$

Posibles resultados del IC*:

a) $\mathrm{Si} \mathrm{IC}^{*}$ es negativo (-40 a -20$)$, su valor relaciona los colores que van desde el azul-violeta al verde profundo.

b) $\mathrm{Si} \mathrm{IC}^{*}$ es negativo (-20 a -2$)$, su valor relaciona los colores que van del verde profundo al verde amarillento.

c) Si IC* está entre $-2 \mathrm{a}+2$, representa el amarillo verdoso.

d) $\mathrm{Si} \mathrm{IC}^{*}$ es positivo $(+2 \mathrm{a}+20)$, se relaciona con los colores que van desde el amarillo pálido al naranja intenso.

e) $\mathrm{Si} \mathrm{IC}^{*}$ es positivo $(+20 \mathrm{a}+40)$, se relaciona con los colores que van desde el naranja intenso al rojo profundo.

Durante el período de almacenamiento poscosecha se midieron las condiciones ambientales (temperatura y humedad relativa) con data-loggers (Hobo ${ }^{\circledR} \mathrm{U10}$ Tem/RH data logger, Onset computer corporation, pocasset, MA USA). 
El diseño experimental de los sistemas de producción fue en bloques al azar con tres repeticiones. Para analizar las variables rendimiento y materia seca se utilizó el Software Estadístico InfoStat y se aplicó ANAVA y test de Duncan. En las evaluaciones poscosecha se utilizó un DCA con tres repeticiones con un nivel de significancia al 0,05 para cada forma de presentación y se ajustó un modelo de medidas repetidas en el tiempo utilizando el enfoque multivariado (11) con el procedimiento MIXED de SAS. Se determinó la estructura de covariancia correspondiente a cada una de las variables analizadas y se incluyeron en el modelo los efectos de los sistemas de producción, la forma de sujeción, el tiempo y la interacción entre ellos, calculando las estadísticas F del ANAVA usual. En los casos en que resultaron interacciones significativas $(p<0,05)$, se consideraron los factores intervinientes y se compararon los niveles de uno de los factores en cada nivel del otro factor, finalizando la comparación estadística en función al tratamiento de menor duración poscosecha.

\section{RESULTADOS Y DISCUSIÓN}

Rendimiento. El rendimiento de un cultivo está estrechamente ligado a la capacidad de acumular biomasa en los órganos que se destinan a la cosecha (materia fresca y seca), por lo tanto, un incremento proporcional de la misma destinada a estos órganos, produce un aumento del rendimiento (17).

En las tres épocas de cultivo se observaron diferencias en el rendimiento entre los sistemas de producción (Figura 2 ), presentando I el mayor valor (otoño $\mathrm{p}<0,01$, invierno $\mathrm{p}<0,05$ y primavera $\mathrm{p}<0,05)$.

Materia Seca. El porcentaje de materia seca en los diferentes órganos de la planta tiene un papel fundamental en la producción de un cultivo y es el resultado final de un conjunto ordenado de procesos metabólicos y de transporte que gobiernan el flujo de asimilados desde la fuente al destino (17). Las actividades involucradas en este proceso

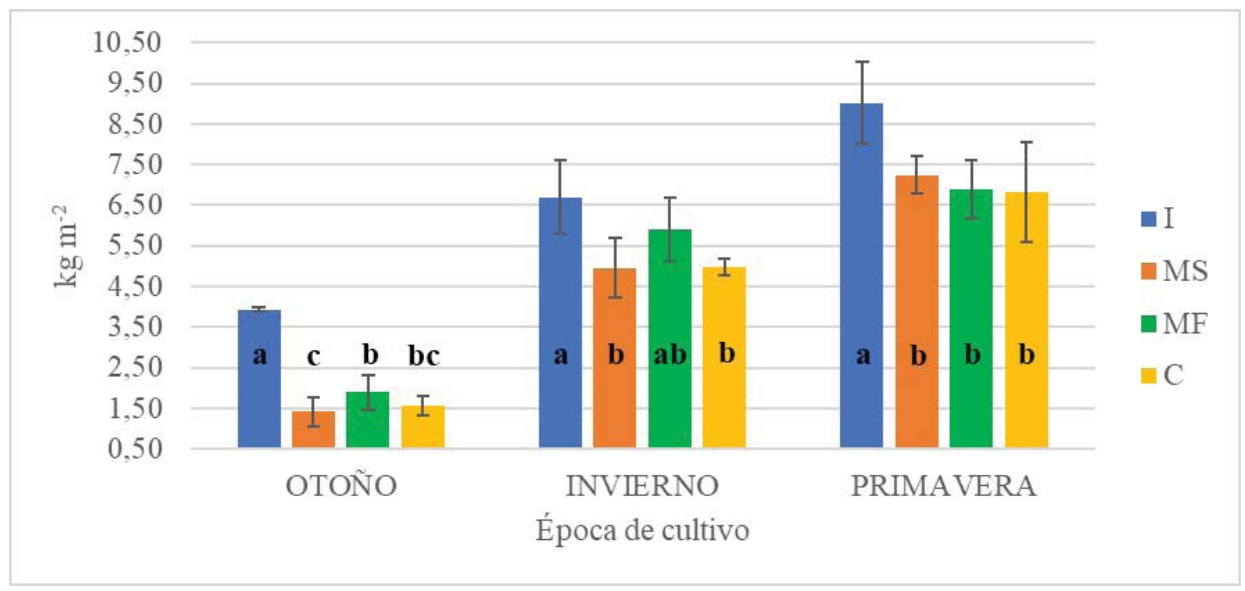

Figura 2: Rendimiento $\left(\mathrm{kg} \mathrm{m}^{-2}\right)$ de los sistemas de producción para cada época de cultivo, desvío estándar y significancia estadística 
no son estáticas y pueden cambiar diariamente y a lo largo del período de desarrollo de la planta (16).

En las tres épocas de cultivo se observaron diferencias en materia seca entre los sistemas de producción presentando MS el mayor valor en otoño $(p<0,05), C$ en invierno y primavera $(p<0,01)$. Los menores valores fueron para I en otoño, I, MS y MF en invierno y MS, MF e I en primavera (Figura 3).

Pérdida de peso por descarte (\%). Si bien fue demostrado que el almacenamiento en cámara frigorífica disminuye el descarte de hortalizas de hoja (4), es importante establecer la influencia que tienen los sistemas de producción sobre el comportamiento poscosecha de acelga en diferentes momentos de cosecha. El sistema de producción en invernadero, además de posibilitar la obtención de mayor rendimiento y calidad (6), permite un mejor comportamiento durante el almacenamiento.

En otoño, el sistema de producción I $(p=0,005)$ y $M F(p=0,0193)$ presenta- ron menores pérdidas de peso por descarte que C. El resultado observado en I es similar al encontrado en rúcula, en condiciones similares (15). En cuanto a días de almacenamiento, los días 3 y 6 $(\mathrm{p}=0,0006)$ presentaron menores pérdidas que el día 9.

En invierno en el día $3(p<0,0001)$ de almacenamiento se registraron menores pérdidas por descarte que el día 9.

En primavera hubo interacción entre días de almacenamiento y forma de sujeción $(\mathrm{p}=0,0040)$ donde la forma de sujeción A presentó pérdidas por descarte menores que $G(p<0,0001)$, en el día 9 (Tabla 2).

Pérdidas de peso por agua (\%). En otoño se registró interacción entre sistemas de producción y días de almacenamiento (Figura 4) donde en el día 3 de almacenamiento el sistema de producción I presentó menores pérdidas que el sistema $C(p=0,0340), \operatorname{MF}(p=0,0118)$ y MS $(p<0,0001)$. En el día 6 de almacenamiento, el sistema de producción MF presentó menores pérdidas que $\mathrm{C}$

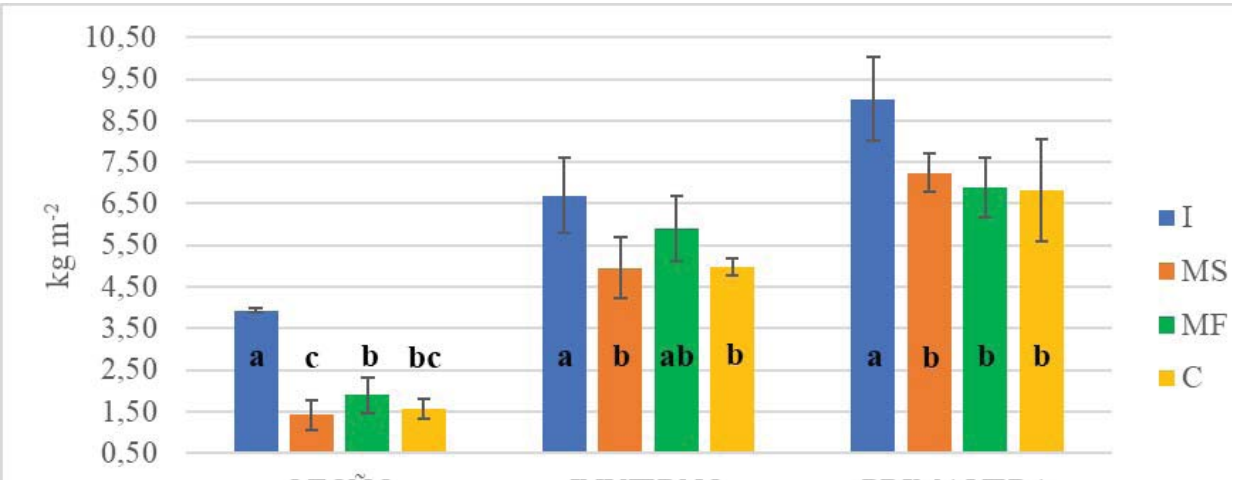

Figura 3: Materia seca (\%) en los diferentes sistemas de producción para cada época de cultivo, desvío estándar y significancia estadística 
Tabla 2: Pérdida de peso por descarte (\%) para sistemas de producción, formas de sujeción y días de almacenamiento en las tres épocas

\begin{tabular}{|c|c|c|c|c|c|c|c|c|c|c|}
\hline \multirow{3}{*}{$\begin{array}{l}\text { Sistemas de } \\
\text { producción }\end{array}$} & \multirow{3}{*}{$\begin{array}{l}\text { Formas de } \\
\text { sujeción }\end{array}$} & \multicolumn{9}{|c|}{ Días de almacenamiento } \\
\hline & & \multicolumn{3}{|c|}{ Otoño } & \multicolumn{3}{|c|}{ Invierno } & \multicolumn{3}{|c|}{ Primavera } \\
\hline & & 3 & 6 & 9 & 3 & 6 & 9 & 3 & 6 & 9 \\
\hline \multirow{2}{*}{ Invernadero } & Atado & 0 & 0 & 2,9 & 0 & 0 & 12,9 & 0 & 0 & 0 \\
\hline & Granel & 0 & 0 & 0 & 0 & 7,9 & 13,7 & 0 & 0 & 9,9 \\
\hline \multirow{2}{*}{ Media Sombra } & Atado & 0 & 0 & 12,4 & 0 & 1,6 & 7,0 & 0 & 4,3 & 0 \\
\hline & Granel & 0 & 0 & 0 & 0 & 9,8 & 3,8 & 0 & 2,6 & 0,9 \\
\hline \multirow{2}{*}{$\begin{array}{c}\text { Manta } \\
\text { Flotante }\end{array}$} & Atado & 0 & 4,6 & 12,2 & 0 & 4,4 & 11,0 & 0 & 5,6 & 3,6 \\
\hline & Granel & 0 & 3,2 & 7,6 & 0 & 4,6 & 10,4 & 0 & 0 & 17,0 \\
\hline \multirow{2}{*}{ Campo } & Atado & 0 & 17,6 & 13,7 & 0 & 10,2 & 19,9 & 0 & 0 & 4,6 \\
\hline & Granel & 0 & 12,4 & 6,1 & 0 & 18,6 & 14,6 & 0 & 7,4 & 10,0 \\
\hline
\end{tabular}

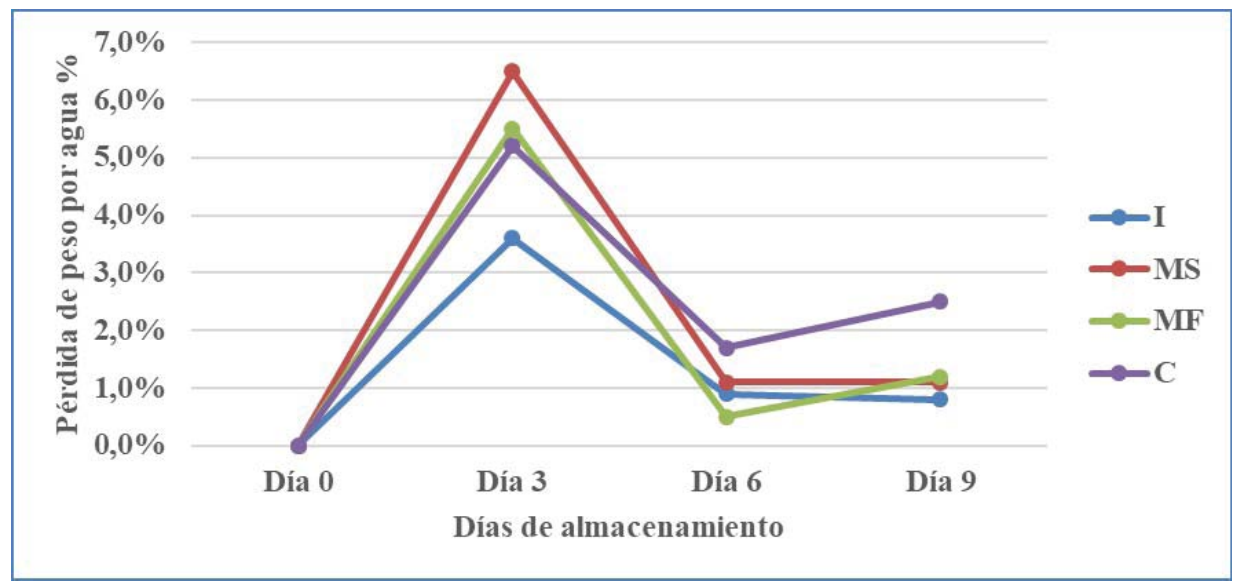

Figura 4: Interacción entre sistemas de producción y días de almacenamiento para pérdida de peso por agua en otoño.

$(\mathrm{p}=0,0118)$. También hubo interacción entre días de almacenamiento y formas de sujeción (Figura 5) donde A presentó menores pérdidas de peso por agua que $\mathrm{G}$ en el día 3 de almacenamiento. Resultados similares fueron encontrados en el cultivo de rúcula, en las mismas condiciones de producción (15).

En invierno no existieron diferencias en ninguno de los factores analizados.
En primavera se encontraron diferencias estadísticas entre días de almacenamiento donde el día 0 presentó menores pérdidas que el día $3(\mathrm{p}<0,0001)$, que el día $6(\mathrm{p}=0,0003)$ y que el día 9 $(p=0,0097)$. El día 9 de almacenamiento obtuvo menores pérdidas de peso por agua que el día 3 ( $\mathrm{p}=0,0097)$ (Tabla 3 ).

La composición de los tejidos vegetales puede verse modificada a causa de 


\section{R. Grasso et al.}

diferentes factores de precosecha tales teracción entre sistemas de producción como el régimen térmico, la irrigación y días de almacenamiento en las tres y condiciones de stress, que afectarían épocas. La interacción se produjo en la pérdida de peso por agua que ocurre todos los días de almacenamiento, pero durante la poscosecha (9). fue variada la respuesta de acuerdo al

Índice de color. Los resultados de ín- sistema de producción en cada época. dice de color en cada época del año se En otoño la MF presentó mayor vapresentan en la Tabla 4. Se observó in- lor (con tendencia al verde amarillento)

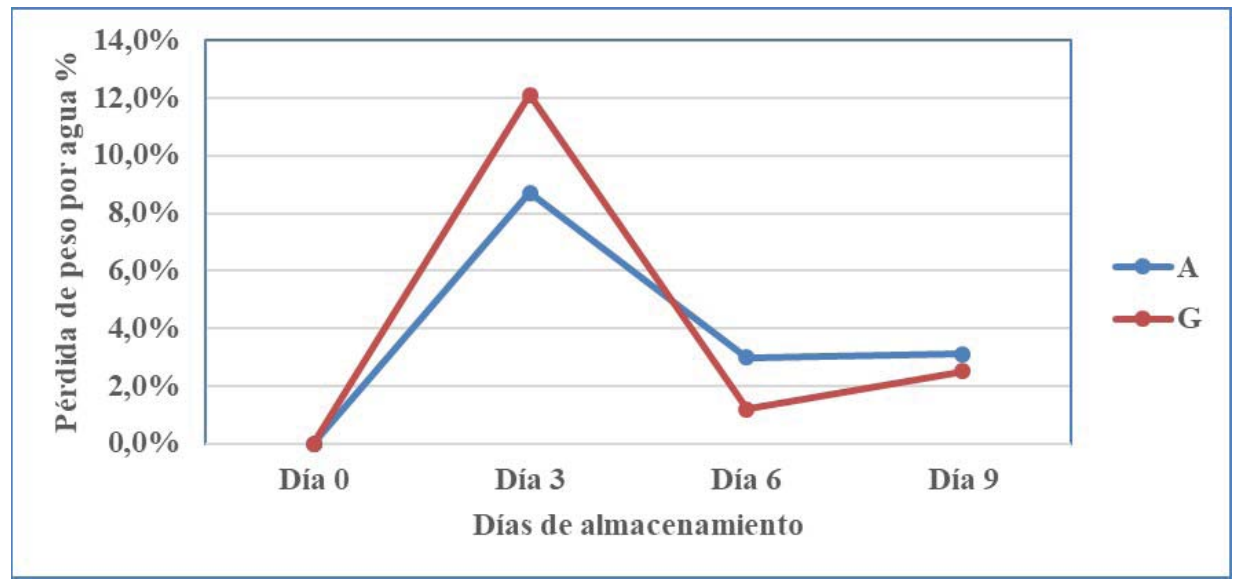

Figura 5: Interacción entre forma de sujeción y días de almacenamiento para pérdida de peso por agua en otoño.

Tabla 3: Pérdida de peso por agua (\%) para sistemas de producción, formas de sujeción y días de almacenamiento en las tres épocas.

\begin{tabular}{|c|c|c|c|c|c|c|c|c|c|c|}
\hline \multirow{3}{*}{$\begin{array}{l}\text { Sistemas de } \\
\text { producción }\end{array}$} & \multirow{3}{*}{$\begin{array}{l}\text { Formas de } \\
\text { sujeción }\end{array}$} & \multicolumn{9}{|c|}{ Días de almacenamiento } \\
\hline & & \multicolumn{3}{|c|}{ Otoño } & \multicolumn{3}{|c|}{ Invierno } & \multicolumn{3}{|c|}{ Primavera } \\
\hline & & 3 & 6 & 9 & 3 & 6 & 9 & 3 & 6 & 9 \\
\hline \multirow{2}{*}{ Invernadero } & Atado & 1,5 & 0,7 & 0,6 & 1,2 & 0,4 & 0,4 & 1,4 & 1,3 & 0,6 \\
\hline & Granel & 2,1 & 0,2 & 0,2 & 0,4 & 0,7 & 2,6 & 1,4 & 1,8 & 1,2 \\
\hline \multirow{2}{*}{ Media Sombra } & Atado & 2,5 & 0,6 & 0,7 & 1,0 & 2,6 & 0,1 & 1,9 & 0,7 & 0,9 \\
\hline & Granel & 4,0 & 0,5 & 0,4 & 0,5 & 1,4 & 0,6 & 1,8 & 1,3 & 0,9 \\
\hline \multirow{2}{*}{$\begin{array}{l}\text { Manta } \\
\text { Flotante }\end{array}$} & Atado & 2,6 & 0,3 & 0,5 & 0,4 & 0,3 & 1,0 & 1,8 & 2,4 & 1,4 \\
\hline & Granel & 2,9 & 0,2 & 0,7 & 0,4 & 0,6 & 0,4 & 1,5 & 1,7 & 1,6 \\
\hline \multirow{2}{*}{ Campo } & Atado & 2,1 & 1,4 & 1,3 & 0,9 & 0,4 & 1,3 & 1,5 & 1,3 & 0,7 \\
\hline & Granel & 3,1 & 0,3 & 1,2 & 1,6 & 0,4 & 0,7 & 5,3 & 0,9 & 0,9 \\
\hline
\end{tabular}


que $\mathrm{C}, \mathrm{MS}$ e I (con tendencia al verde profundo). Este comportamiento se observó en todas las mediciones durante los días de almacenamiento ( $\mathrm{p}=0,0190$ entre sistemas $\mathrm{x}$ días).

En invierno $\mathrm{C}$ registró mayor valor que I, MS y MF en el día 0. En los 3 días siguientes de medición poscosecha, se observó mayor valor de $\mathrm{C}$ respecto a MS, I y MF $(p=0,0063)$. Es decir que durante todo el almacenamiento el sistema $\mathrm{C}$ presentó valores de color verde menos profundo respecto a MF, con valores intermedios para los demás sistemas.

En primavera $(p=0,0063)$ el mayor valor lo obtuvo I, seguido por MS, MF y $\mathrm{C}$, en los días 0 y 3 de almacenamiento, es decir que la acelga cultivada bajo los sistemas protegidos presentó color verde menos profundo. En cambio, en las dos mediciones siguientes, la evolución del color fue diferente. Si bien el I presentó el mayor valor, en el día 6 fue seguido por C, MF y MS y en el día 9 de almacenamiento fue seguido por MS, C y MF.

\section{CONCLUSIONES}

En las tres épocas de cultivo de acelga, se obtuvo el mayor rendimiento y menor contenido de materia seca en el sistema de producción invernadero.

Las diferencias entre sistemas de producción para la variable pérdida de peso por descarte, solo se registraron en otoño, donde los sistemas protegidos invernadero y manta flotante disminuyeron las mismas. A medida que transcurren los días de almacenamiento, en otoño e invierno, aumentan dichas pérdidas. La forma de sujeción atado disminuye el descarte hacia el final del almacenamiento en primavera. Los sistemas de producción invernadero y manta flotante disminuyen las pérdidas de peso por agua en los días 3 y 6 de almacenamiento en otoño. En primavera la pérdida de peso por agua aumenta en la etapa inicial del almacenamiento disminuyendo hacia el final del mismo. La acelga obtuvo un color

Tabla 4: Índice de color para sistemas de producción, formas de sujeción y días de almacenamiento en las tres épocas.

\begin{tabular}{|c|c|c|c|c|c|c|c|c|c|c|c|c|c|}
\hline \multirow{3}{*}{$\begin{array}{l}\text { Sistemas de } \\
\text { producción }\end{array}$} & \multirow{3}{*}{$\begin{array}{l}\text { Formas } \\
\text { de } \\
\text { sujeción }\end{array}$} & \multicolumn{12}{|c|}{ Días de almacenamiento } \\
\hline & & \multicolumn{4}{|c|}{ Otoño } & \multicolumn{4}{|c|}{ Invierno } & \multicolumn{4}{|c|}{ Primavera } \\
\hline & & 0 & 3 & 6 & 9 & 0 & 3 & 6 & 9 & 0 & 3 & 6 & 9 \\
\hline \multirow{2}{*}{ Invernadero } & Atado & $-25,5$ & $-24,6$ & $-25,1$ & $-23,1$ & $-23,2$ & $-23,5$ & $-23,1$ & $-24,2$ & $-18,8$ & $-18,9$ & $-19,2$ & $-18,3$ \\
\hline & Granel & $-29,1$ & $-27,9$ & $-28,0$ & $-25,7$ & $-24,1$ & $-24,2$ & $-23,8$ & $-23,0$ & $-17,5$ & $-18,2$ & $-18,1$ & $-18,6$ \\
\hline \multirow{2}{*}{$\begin{array}{l}\text { Media } \\
\text { Sombra }\end{array}$} & Atado & $-20,1$ & $-18,9$ & $-19,7$ & $-17,9$ & $-23,5$ & $-22,9$ & $-22,2$ & $-22,2$ & $-20,9$ & $-21,3$ & $-22,1$ & $-20,3$ \\
\hline & Granel & $-23,2$ & $-23,2$ & $-22,8$ & $-21,6$ & $\mid-24,9$ & $-24,4$ & $-21,4$ & $-21,1$ & $-21,0$ & $-21,8$ & $-22,5$ & $-20,9$ \\
\hline \multirow{2}{*}{$\begin{array}{l}\text { Manta } \\
\text { Flotante }\end{array}$} & Atado & $-17,3$ & $-18,0$ & $-17,2$ & $-17,0$ & $-29,6$ & $-26,9$ & $-25,9$ & $-23,7$ & $-21,2$ & $-20,8$ & $-20,7$ & $-25,2$ \\
\hline & Granel & $-18,9$ & $-19,3$ & $-19,9$ & $-18,7$ & $-28,6$ & $-28,3$ & $-25,4$ & $-24,2$ & $-21,9$ & $-22,5$ & $-22,0$ & $-22,7$ \\
\hline \multirow{2}{*}{ Campo } & Atado & $-20,3$ & $-20,3$ & $-21,2$ & $-20,2$ & $-21,5$ & $-19,9$ & $-19,7$ & $-19,2$ & $-21,4$ & $-22,4$ & $-20,7$ & $-21,5$ \\
\hline & Granel & $-20,8$ & $-21,4$ & $-21,3$ & $-20,2$ & $-21,9$ & $-20,7$ & $-20,6$ & $-19,9$ & $-21,9$ & $-21,0$ & $-21,7$ & $-21,0$ \\
\hline
\end{tabular}


con tendencia hacia el verde amarillento en el sistema de producción manta flotante en otoño, campo en invierno e invernadero en primavera, en todos los días de almacenamiento.

\section{BIBLIOGRAFÍA}

1.- BOUZO, C. A.; FAVARO, J. C.; PILATTI, R. A. \& SCAGLIA, E. M. 2005. Cinturón Hortícola de Santa Fe: Descripción de la zona y situación Actual. Revista FAVE - Ciencias Agrarias 4 (1-2).

2.- CENSO NACIONAL AGROPECUARIO. 2002. INDEC. Instituto Nacional de Estadísticas y Censos. http://www.indec.gob.ar/index_agropecuario.asp. Acceso: 01/12/2017.

3.- FERRATTO, J.; MONDINO, M.; GRASSO, R.; ORTIZ MACKINSON, M.; LONGO, A.; CARRANCIO, L.; FIRPO, I.; ROTONDO, R.; ZEMBO, J.; CASTRO, G.; GARCÍA, M.; RODRIGUEZ, M.; IRIBARREN, M. 2010. Buenas Prácticas Agrícolas para la agricultura familiar. Cadena de las principales hortalizas de hojas en Argentina. FAO pp. 535.

4.- FIRPO, I.; ROTONDO, R.; FERRATTO, J.; GRASSO, R.; TREVISAN, A.; RODRIGUEZ, M. 2012. Incidencia del ambiente y la revitalización en las pérdidas poscosecha de hortalizas de hojas, fruto y raíz. Ciencias Agronómicas. Revista de la Facultad de Ciencias Agrarias. UNR. N ${ }^{\circ}$ XX Año 12, p. 007-013. ISSN N ${ }^{\circ}$ 1853-4333, ISSN ON-LINE: 2250-8872.
5.- GRASSO, R.; MONDINO, M.C.; ORTIZ MACKINSON, M.; VITA LARRIEU, E.; LONGO, A.; FERRATTO, J.A. 2013. Censo 2012 del Cinturón Hortícola de Rosario. Ajuste del Diagnóstico Agronómico de necesidades y estrategias de intervención del Proyecto Hortícola de Rosario (2013/2018). Publicación Miscelánea № 50. Estación Experimental Agropecuaria Oliveros. INTA. Santa Fe. Junio 2013. ISSN. 0326-256. 31 pp.

6.- GRASSO, R.; ORTIZ MACKINSON, M.; ROTONDO, R.; MONDINO, M.C.; CALANI, P.; FIRPO, I.; BALABAN, D.; VITA LARREAU, E. 2017. Productividad de rúcula (Eruca sativa Mill.) en diferentes sistemas productivos. Agromensajes 47:3035. Facultad de Ciencias Agrarias, UNR.

7.- НАMАMOTO, Н. 1996. Effect of non-woven rowcover on plant environment and growth. Japan Agricultural Research Quarterly, 30 (1), p. 49-53.

8.- HANADA, T. 1991. The effect of mulching and row covers on vegetable production. Chugoku Agr. Exp. Stn. Ueno 200, Ayabe city, Kyoto Pref. 623, Japan. http://www.fftc.agnet. org/htmlarea_file/library/20110801145616/ eb332.pdf. Acceso: 01/12/2017.

9.- HELYES, L. \& LUGASI, A. 2006. Formation of certain compounds having technological and nutritional importance in tomato fruits during maturation. Acta alimentaria, 35 (2): 183-93

10.- JENSEN, M. H.; MALTER, A. J. 1995. Protected Agriculture: A Global Review, Vol 23-253. World Bank Publications. Technical Paper Number 253. World Bank Washington DC. USA 
11.- KHATTREE, R.; NAIK, D. N. 1999. Applied Multivariate Statistics With. SAS Software. Second Edición. SAS Institute Inc. Cary, NC, USA. Pag. 338.

12.- MASHEGO, D. 2001. The production of vegetable crops under protection for Small-scale farming situations. Department of plant production and soil science Faculty of natural and agricultural sciences University of Pretoria. https://repository.up.ac. $\mathrm{za} /$ dspace/bitstream/handle/2263/26198/ dissertation.pdf? sequence $=1 \&$ amp; sAllowed=y. $\quad$ Acceso: 01/12/2017.

13.- MONDINO, M.C; FERRATTO, J.; FIRPO, I. T.; ROTONDO, R.; ORTIZ MACKINSON, M.; GRASSO, R.; CALANI, P.; LONGO, A. 2007. Pérdidas poscosecha de lechuga, en la región de Rosario, Argentina. Horticultura Argentina (26) No 60 p17-24.

14.- OLLE, M.; BENDER, I. 2010. The effect of non-woven fleece on the yield and production characteristics of vegetables. Jogeva Plant Breeding Institute, J. Aamisepa 1 Jõgevaalevik 48309, Estonia.

15.- ORTIZ MACKINSON, M.; GRASSO, R.; ROTONDO, R.; CALANI, P.; MONDINO, M.; BALABAN, D.; VITA LARREAU, E.; MONTIAN, G.; BARBONA, I. 2017. Efecto de distintos sistemas de producción y formas de sujeción sobre las pérdidas poscosecha de rúcula. Horticultura Argentina 36 (91): Sep.-Dic. 2017. ISSN de la edición on line 1851-9342.
16.- PATRICK, J. W. 1988 Assimilate partitioning in relation to crop productivity. HortScience, v.23, p.33-40.

17.- PEIL, R. M. \& GÁLVEZ, J. L.. 2005. Reparto de materia seca como factor determinante de la producción de las hortalizas de fruto cultivadas en invernadero. R. bras. Agrociência, vol.11, n. 1, p. 05-11, jan-abr, 2005.

18.- ROMOJARO ALMELA, F.; FLORES F. B.; EGEA, M. I. SÁNCHEZ BEL, PALOMA MARTÍNEZ, M C; RIBAS, F. CABELLO M. J. 2007. Factores precosecha que afectan a la calidad de frutas y hortalizas Phytoma ISSN 1131-8988, Nº 189, pág 43-50.

19.- ROTONDO R.; FIRPO I.T.; FERRATTO J. A.; DÍAZ, B.M.; VIGNAROLI, L. 2000. Efecto del acolchado del suelo con paja y mantas flotantes, sobre la productividad de lechuga (Lactuca sativa L.), en otoño. XXII Congreso Argentino de Horticultura, X Latinoamericano y III Iberoamericano. 2000. 20.- VIGNONI, L. A., CÉSARI, R. M., FORTE, M., \& MIRÁBILE, M. L. 2006. Determinación de índice de color en ajo picado. Información tecnológica, 17(6), 63-67.

21.- WESTLAND, S.; GRAHAM, C.; ADDISON, S.; SHARROTT, P.; RIGG, B. 2001. Effect of sleeve color and background color on change in color assessments. Coloration Technology 117 (3), 123-126. 